\title{
Prediction of moving target behavior optimization based on Dirichlet model
}

\author{
Chen Zheng ${ }^{1,2}$, Zhang Heng-yi ${ }^{3}$ \\ 1. Hunan Institute of Technology, Computer and Information Science, Hengyang, Hunan 421002 \\ 2. Computer and Information Science, Southwest University, Beibei, Chongqing, 400715 \\ 3. Business College, Central South University of Forestry and Technology, Changsha, Hunan, \\ 410004
}

Keywords: Dirichlet model; text feature weighting; abnormal behavior prediction; video image mining

Abstract. With the rapid development of science and technology, video monitoring has entered in to all walks of life, how to use computer for video image processing, analysis and understanding is an important research field of computer image mining. How to combine the computer technology with video monitoring system is the perfect combination of modern monitoring system development trend, has far-reaching significance.

\section{Modeling and weighted optimization Dirichlet}

\section{Dirichlet Modeling and Optimization.}

dual-HDP model for discrete data sets (such as document collection) probabilistic modeling growth model is a four-Bayesian model, a brief description of the document, to retain the essence of statistical information to help efficiently handle massive documentation set. Consider $\mathrm{k}$ themes document $\mathrm{d}_{\mathrm{k}}$ in $\mathrm{i}$-th word $\mathrm{w}_{\mathrm{i}}$ of the probability can be expressed as follows:

$$
P\left(w_{i}\right)=\sum_{l=1}^{C} P\left(c_{k}=l\right) \sum_{j=1}^{T} P\left(w_{i} \mid z_{i}=j\right) P\left(z_{i}=j \mid c_{k}=l\right)
$$

Which, zi, ck is the latent variable, zi denote the i-th vocabulary wi taken from the subject, ck $\mathrm{k}$ denotes the set of documents belonging to the document. $P(w i \mid z i=j)$ is part of the theme words wi j probability, $\mathrm{P}(\mathrm{zi}=\mathrm{j} \mid \mathrm{ck}=\mathrm{l})$ gives the probability that the document belongs to the documentation set $\mathrm{dk} \mathrm{j}, \mathrm{l}$ theme, $\mathrm{P}(\mathrm{ck}=\mathrm{l})$ indicates that the document $\mathrm{d} \mathrm{l}$ the introduction belong documentation set. J-th theme is expressed as a polynomial distributed $\Psi$ wji vocabulary words of $v=P(w i \mid z i=j)$, expressed as $\mathrm{K}$ cryptic text on the subject of random mixing. So the probability of text $\mathrm{d}$ in "happen" Words $\mathrm{w}$ as: $\theta \mathrm{jd}=\mathrm{P}(\mathrm{Zi}=\mathrm{j})$, then the probability of words appearing in $\mathrm{W}$ d for text $P(w \mid d)=\sum_{j=1}^{T} \varphi_{w}^{j} \cdot \theta_{j}^{d}$

Text feature weighting methods commonly used word $w$ of information gain, the text features expected cross entropy, mutual information text feature, $\mathrm{x} 2$ statistics, odds ratio (Odds ratio) and the document word frequency - inverted text method (TF-IDF) and so on. In addition to TF-IDF, other methods are required to consider the class label is applied to supervised learning feature selection, whereas LDA theme unsupervised learning is the process of dividing the word in the text of unsupervised learning, common TF-IDF carried out feature weighting. For the TF-IDF weighting assumption is: Many of the number of occurrences of words in a text, the number of occurrences will be much, and vice versa in the other similar text. But if such a distribution is not satisfied, the weighted effect is not ideal as for diagnostic data, each word appears only once, that is a TF in each sample, if the weight is inversely proportional to the right leads to the word frequency and weighted by TF -IDF It does not meet the common sense. The purpose here is to reduce the weighting of high-frequency words without increasing the weight of heavy low-frequency words right. 
In this paper, a Gaussian function of term weighting. The purpose is to reduce the high-frequency words weight, improve the IF word weights. This article assumes that appear in the same document, the same words have the same weight, in the words of the same subject, the greater the distribution ratios of the word In the greater importance of the topic.

Firstly, the formula for the Gaussian word document m weighted for am $=\exp [-(\mathrm{fm}-\mathrm{fi}) 2 / 2 \sigma 2]$ $+1$

Where: Varianc

$$
\sigma^{2}=\frac{\sum_{m=1}^{V}\left(f_{m}-f_{i}\right)^{2}}{V-1}
$$

, $f_{m} m$ is the number of words in the word frequency, $f_{i}$ is the number of word frequency count word frequency centered word of i. Back 1 is to ensure that the ratio between am not too large, it can be seen from the above equation, it is argument interval $(1,2)$.

In order to keep the total word word weighted total number of data sets of the same number and weighted before, using the equation for processing, where $n$ is the total number of words in the documentation set:

$$
W_{m}=\frac{n}{\left(\sum_{m=1}^{V}\left(f_{m} * a_{m}\right)\right)}
$$

The weighting coefficients introduced dual-hdp above model, then a characteristic word w is generated not only with the sampling formula $\Phi$ tkt related, but also with the right word in the document features a heavy weight $(\mathrm{t})$ related to, namely:

$$
p(w \mid \theta, \varphi)=\frac{\text { weight }(w) * \varphi_{k}^{t}}{\sum_{w \in V} \text { weight }(w)}
$$

In this paper, the right to feature the word heavy weight (t) with a sampling formula $\Phi$ tk combined to different characteristic words assign different weights at different topic, change probability model generation feature words, combined with equation (3) (4) obtained and the formula (5) a Gibbs sampling new formula derived parameters

$$
\begin{gathered}
p\left(z_{i}=k \mid z_{-i}, w, \alpha, \beta\right) \propto p\left(z_{i}=k, w_{i}=t \mid z_{-i}, w_{-i}, \alpha, \beta\right) \propto \\
\frac{w e i g h t(w)^{*} n_{k}^{w}+\beta}{\sum_{t=1}^{v}\left(\text { weight }(t)^{*} n_{k}^{t}+\beta\right)} * \frac{\text { weight }(w)^{*} n_{m}^{k}+\alpha}{\sum_{j=1}^{K}\left(\text { weight }(w)^{*} n_{m}^{j}+a\right)}
\end{gathered}
$$

\section{Experimental Verification}

Experimental data:(Actual data) a foreign city crossroads 15:15 surveillance video

Experimental environment: Intel Pentium CORE II E8400 processor, DDR-II 4 GB of memory, Windows XP operating system. Algorithms using JAVA language, matlab virtual platform.

\section{Single-target motion detection.}

In this paper, the experimental data, the lowest point of the feature displays the results of clustering, on behalf of its single goal of motor behavior trajectories shown in Figure1 


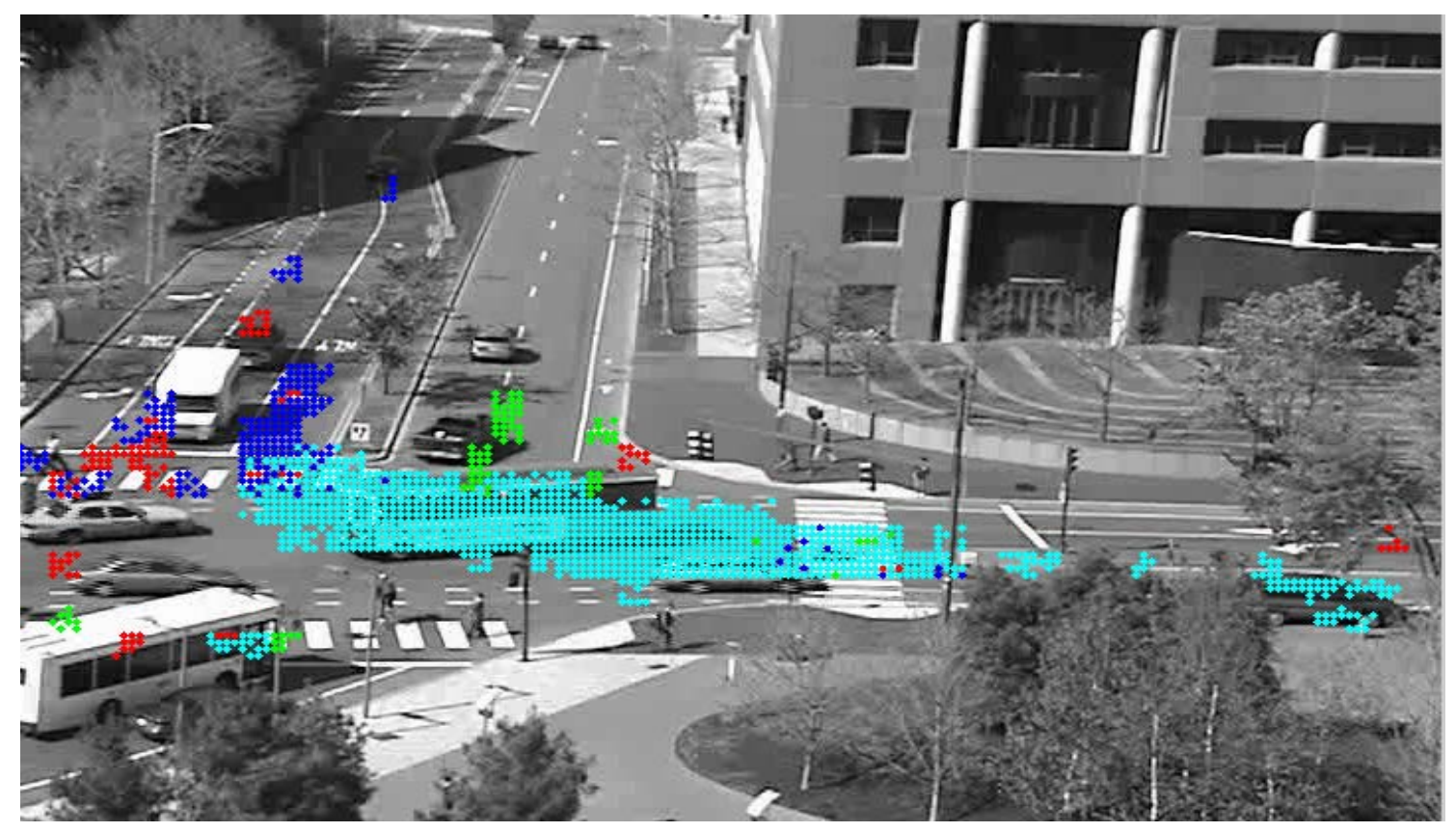

Figure 1 Single-target trajectory monitoring

Points labeled in FIG. 2 for a pixel classified into the class, different colors are represented by a corresponding point in different directions. Dark blue pixels from the top down movement, left to right direction on behalf of blue and red points the direction of movement from right to left, green represents the point from the bottom up movement. This category represents a major downward movement of the vehicle and then turn left.

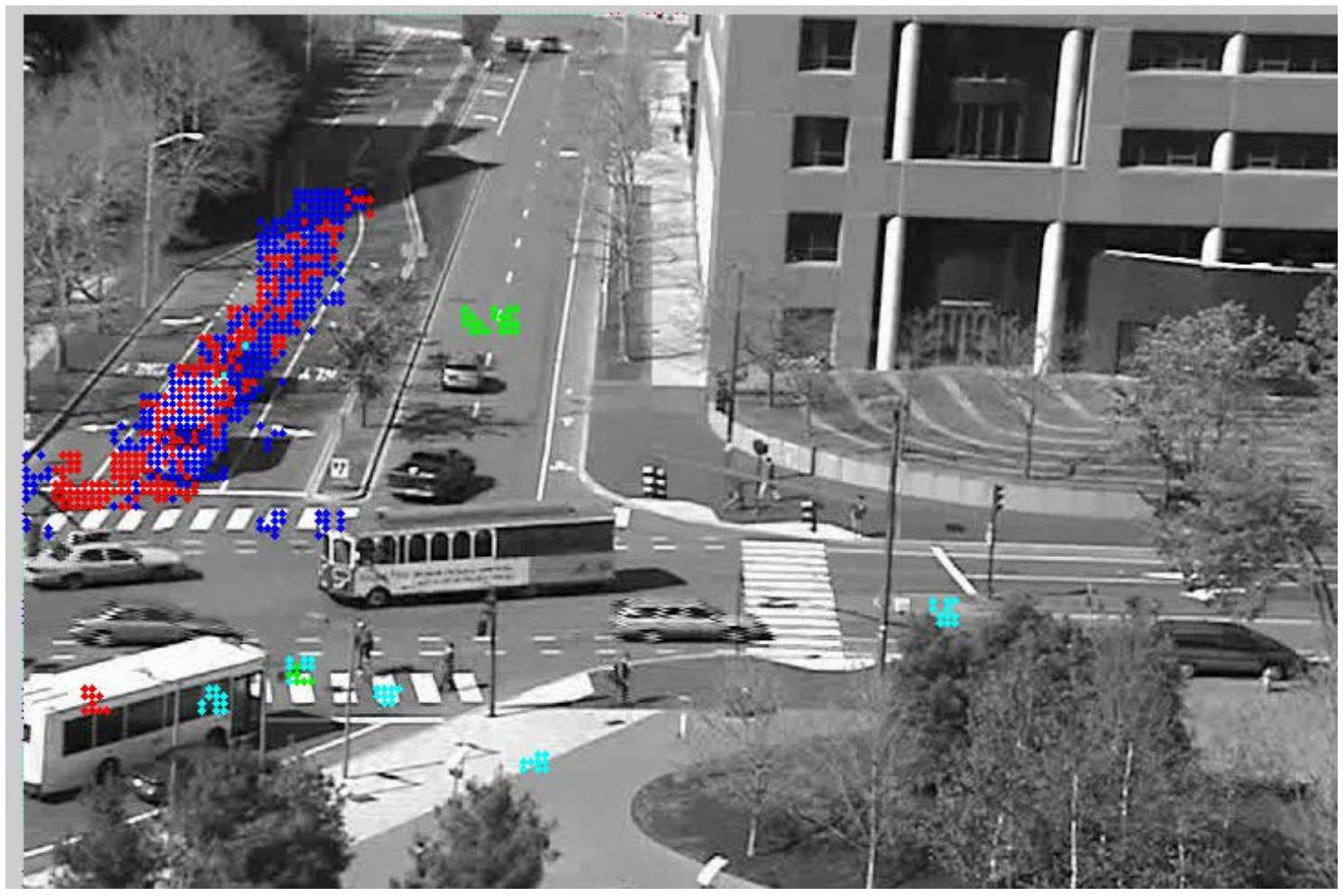

Figure 2 single-target trajectory monitoring

\section{Interactive multi-target action probability forecast.}

Video surveillance system in this article, the second layer clustering results represent the interactive multi-target movement in video traffic junctions, the second layer of each category, that is, the traffic lights under different stages, each of the first occurrence probability distribution layer of single-target movement. 


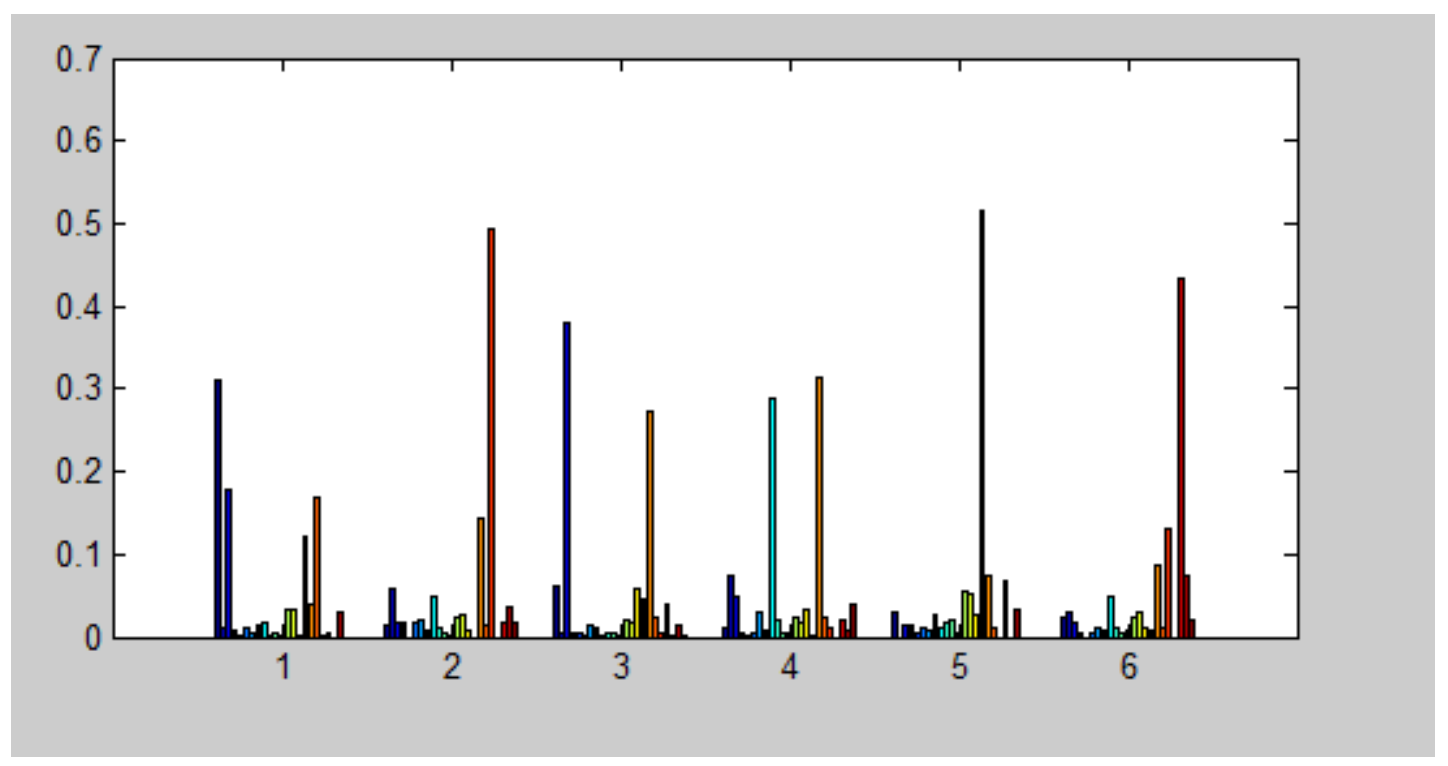

Figure 3 Multi-target trajectory prediction probability distribution

Figure 3 is a model of the probability of different clusters of the second layer histogram can be seen from the figure, the interaction of different actions, each containing a single target very different sports.

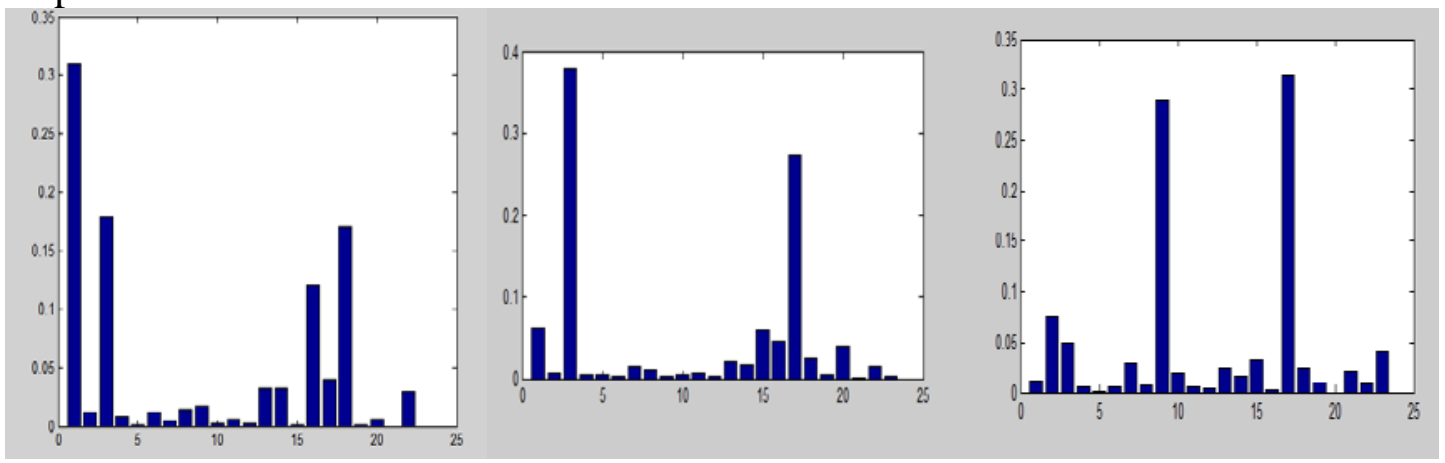

Figure 4 Multi-objective clustering probability histogram

Figure 4 shows three clusters detailed probability histogram, the abscissa represents the 23 categories generated by the underlying cluster, the ordinate represents the percentage of the total number of different categories of clustering.

\section{Anomaly Detection.}

Video surveillance system, abnormal behavior for two, one is a single target abnormal behavior, such as pedestrians crossing the road does not go the sidewalk. The second is the normal behavior of a single target interaction occurs in the wrong action, such as red light running vehicles.

\section{Conclusion}

In this paper, the problem of image mining surveillance images of the actual road intersection as a starting point for intelligent video surveillance of the moving object recognition problem, first proposed the weighted hierarchical Bayesian model; according to some details of the control system and the basic structure of a priori information points establish procedures related models, when some parameters can not be determined a priori need to set up another super-parameter, which is a combination of a priori stratification. Bayesian analysis is then stratified prior distribution hypothesis for the model to optimize debug their excessive influence on the estimation results, and enhance the robustness of estimates, the optimizer Dirichlet model with a strong practical. The main contribution of this paper is to: 1) for intelligent video surveillance to identify the moving target problem, a weighted hierarchical Bayesian model. The core idea is to select a priori hierarchical prior distribution. 2) the hierarchical Bayesian analysis of prior distribution hypothesis for the 
model, helping to eliminate the prior distribution over the estimated impact on the outcome, and enhance the robustness of the estimate, so that the model has strong applicability.

\section{References}

[1]Lipton A, Kanade T, Fujiyoshi H, et al. A system for video surveillance and monitoring[M]. Pittsburg: Carnegie Mellon University, the Robotics Institute, 2000.

[2]Johnson A Y, Bobick A F. A multi-view method for gait recognition using static body parameters[C]//Audio-and Video-Based Biometric Person Authentication. Springer Berlin Heidelberg, 2001: 301-311.

[3] Matsuyama T. Cooperative distributed vision[J]. Knowledge Based Computer Vision, 1998: 8.

[4] Wang X, Ma X, Grimson W E L. Unsupervised activity perception in crowded and complicated scenes using hierarchical bayesian models[J].Pattern Analysis and Machine Intelligence, IEEE Transactions on, 2009, 31(3): 539-555.

[5] Zhang Haiqing. Target motion detection and tracking research computer intelligent video surveillance system [D]. China University of Science and Technology master's degree thesis, 2007.

[6] Liu Leping, Pinson right, any Akemi. Disability rate is small domain estimation method based on hierarchical Bayesian analysis [J]. Statistical Research, 2010,27 (3): 83-88.

[7] Kong Xiaodong. Intelligent video surveillance technology research [D]. Shanghai Jiaotong University master's degree thesis, 2008.

About the Author: Chen Zheng (1988-), male, Hunan Changning, master candidate in Information Science School in Hunan Institute of Technology. Research direction: Computer and Information Science.

Corresponding author: Zhang Heng-yi (1991-), male, Hunan Jishou, master candidate in Business School in Central South University of Forestry and Technology. Research direction: Forestry ecological and economic management. 